\title{
LOS MECANISMOS DE MODALIZACIÓN EN LAS ORACIONES CONDICIONALES NO REALES: DEL LATÍN A LOS ROMANCES (CON ESPECIAL ATENCIÓN AL CASTELLANO)
}

Wiaczesław Nowikow ${ }^{1}$

https://doi.org/10.18778/8220-421-6.04

\begin{abstract}
The aim of this paper is to analyze the mechanism of modalization in conditional sentences during the evolution from Latin to Romance languages. The analysis is realized with special attention to Spanish. The modal modification of the transmitted contents is connected with two factors (mood and tense): 1. The modal value of the Subjunctive mood and 2. The temporal translocation of the Latin and Romance tenses. Some Romance languages (e.g. Spanish, Portuguese and Italian) represent mostly the first solution. Others languages (e.g. French, Occitan and Catalan) select exclusively or principally the second possibility. Nevertheless, the temporal translocation connected with the change of the modal contents is possible in all Romance languages.
\end{abstract}

Keywords: Latin, Romance languages, Spanish, mood, tense, conditional sentences, typological approach.

Palabras clave: latín, lenguas románicas, español, modo, tiempo, oraciones condicionales, enfoque tipológico.

1 Universidad de Łódź, Facultad de Filología, Departamento de Filología Española, c/ Pomorska 171/173, Łódź, Polonia. 
1.

\section{Introducción}

En el presente trabajo vamos a analizar los mecanismos de modalización en los esquemas condicionales no reales en las lenguas romances, siendo nuestro punto de partida el latín y dedicando especial atención al español. Metodológicamente, el análisis se sitúa dentro de la corriente tipológica. Esto quiere decir que lo que nos interesa en primer lugar es establecer las diferencias tipológico-estructurales, por un lado, entre el latín y las lenguas románicas y, por otro, entre diversas variedades romances. De modo que en esta ocasión no vamos a abordar cuestiones relacionadas con los esquemas periféricos propios de algunas variedades diastráticas y/o diatópicas, preferencias estilístico-discursivas, etc. Nuestro análisis girará en torno a tres cuestiones básicas:

a) las diferencias de índole tipológica entre las lenguas con respecto a los mecanismos de modalización del contenido proposicional;

b) las reparticiones formal y semántica de los esquemas condicionales no reales y los tipos de mecanismos de modalización en el latín;

c) la evolución de los esquemas condicionales no reales en las lenguas románicas y el establecimiento de tres modelos tipológicos básicos en función de los mecanismos de modalización.

\section{2.}

\section{La modalización del contenido proposicional}

Se trata de un procedimiento universal que se da en varias lenguas entre dos secuencias de un enunciado con la estructura argumentativo-predicativa explícita actualizada a través de una oración compleja que puede ser representada, por ejemplo, mediante el siguiente encadenamiento sintáctico-semántico (véase, a este respecto, Nowikow, 2013: 67-68 y 2018: 105): 
(1) Lo haces $\rightarrow$

(2) Quiero / dudo / estoy contento $\rightarrow$

(3) Quiero / dudo / estoy contento (de) que lo hagas.

Como se ve, en este caso la modalización se lleva a cabo por medio de una operación que consiste en el cambio gramatical de tipo modal \{indicativo (IND) $\rightarrow$ subjuntivo (SUBJ)\}:

(4) lo haces IND $\rightarrow$ lo hagas SUBJ.

Es obvio que la modalización puede realizarse también en una oración simple a través del empleo de recursos léxicos y/o gramaticales (p. ej., adverbio dubitativo + IND / SUBJ: quizá es / sea nuestro amigo).

No obstante, lo que nos interesa en esta ocasión es llamar la atención a la existencia de determinados tipos de modalización basados en los fenómenos y elementos gramaticales cuyas combinaciones permiten distinguir dos clases de lenguas básicas.

\section{3.}

\section{Lenguas "DT + MS" vs. lenguas "MH + NM"}

Nos referimos a dos tipos de lenguas que modalizan el contenido proposicional mediante los procedimientos que consisten en la combinación de los siguientes factores (cfr., p. ej., Nowikow, 2013: 68-70 o 2017: 19-20):

1) desplazamiento (dislocación) temporal (DT) + modo subjuntivo (MS) y

2) modo hipotético $\mathrm{MH}+$ nexos modalizados (NM).

A las primeras pertenece, por ejemplo, el español y a las segundas el polaco. En español se da tanto el desplazamiento temporal (los tiempos verbales sustituyen la relación temporal primaria o recta por la secundaria o dislocada, cfr., a este respecto, también Rojo, 1974; Rojo, Veiga, 1999; Veiga, 2012; Nowikow, 2017: 144-148; Nowikow, 2018b):

(5) ¿Qué hora es? - Serán las 2.

(6) De buena gana me tomaría un mosto.

como el empleo obligatorio u optativo del modo subjuntivo en determinadas distribuciones sintáctico-semánticas: 
(7) Séque es así vs. Dudo que sea así.

(8) Celebraremos un simposio que interesará / interese a muchos lingüistas.

(9) Aunque llueve/llueva, iremos de excursión.

El desplazamiento temporal se ve acompañado por la aparición de los contenidos modales de 'incertidumbre' (5) y 'no realidad' (6), mientras que el contenido funcional del modo subjuntivo es la 'no aserción' $(7,8,9)$ que consiste, grosso modo, en la falta de compromiso con la veracidad de lo que se enuncia (el llamado dictum).

En cambio, el sistema verbal del polaco cuenta con una forma que representa el modo hipotético eslavo formado mediante la combinación del participio pasado activo en - $l$ - y la palabra auxiliar bim $\iota$ creada a base de la raíz ${ }^{\star} b h u$ y del morfo optativo $-i-:{ }^{\star} b h u+-i-=b w i \rightarrow b i \rightarrow$ by: dałby (3a .pers. sing. masc. de $d a c ́$ [esp. dar]): $d a+\nmid+b y$ (cfr. Nowikow, 1991, 2013 y 2017: 29). Por ejemplo:

(10) Poszedłbym [1ª.pers. sing. masc.] do kina (posposición).

(11) Do kina bym poszedt [1 ${ }^{\text {a }}$.pers. sing. masc.] (preposición). (esp. Iría al cine).

(12) Zrobiłabyś to jutro (2a. pers. sing. fem.).

(esp. Lo harías mañana).

(13) Chcę, żebyś to zrobiła jutro (2a ${ }^{a}$ pers. sing. fem.).

(esp. Quiero que lo hagas mañana).

Por otro lado, cabe subrayar que el polaco dispone de nexos modalizados de tipo żebyś (cfr. el ejemplo (13), supra) que se compone de los morfos że (esp. que) + byś (by [el morfo del modo hipotético] + ś [el morfo de la $2^{\mathrm{a}}$. pers.). Los nexos sintéticos modalizados se dan en diferentes tipos de oraciones complejas, por ejemplo, condicionales (jeśliby / gdyby, esp. si), concesivas (chociażby, esp. aunque), finales ( $\dot{z}$ eby /aby, esp. para que), sustantivas (żeby, esp.que), etc.

Cabe señalar que, a diferencia del español, el desplazamiento temporal se da en polaco con muy pocos verbos y se ve sometido a varias restricciones sintáctico-semánticas:

(14) Ile lat ma Jan? - Będzie miat (ze) 20.

(esp. ¿Cuántos años tiene Juan? - Tendrá 20.) 


\section{4.}

\section{Los esquemas condicionales del latín con valor de 'no realidad'}

Como es sabido, las oraciones condicionales del latín se caracterizan, tradicionalmente, por una repartición tripartita al distinguir entre esquemas (véase, p. ej., Nutting, 1926; Safarewicz, 1950; Bassols de Climent, 1976; Stefański, 1987; Altieri Megale, 1988):

1) reales: $s i+$ indicativo (p.ej., si habeo, do; los tiempos Presente, Futuro I/II, Perfecto);

2) potenciales: $s i+$ subjuntivo potencial (p.ej., si habeam, dem; los tiempos Presente, Perfecto o Imperfecto: si haberem, darem) $y$

3) no reales (irreales): $s i+$ subjuntivo irreal.

Dentro de la clase de esquemas condicionales no reales se distinguen los:

a) de simultaneidad / posterioridad: si haberem, darem (imperfecto de subjuntivo);

b) de anterioridad: si habuissem, dedissem (pluscuamperfecto de subjuntivo).

No obstante, ya en la epoca arcaica (Plauto, s. III-II a. de J.C.) se daba la sustitución de cantarem por cantavissem cuya difusión aumentó en el periodo clásico:

(15) non fugisset hoc graecos homines, si ita necesse esse arbitrari essent (Cic.) (Nowikow, 1995: 169; todos los ejemplos latinos provienen de este estudio).

El cambio fue iniciado por los verbos modales (debuissem, potuissem, voluissem) y finalmente hacia el $\mathrm{s}$. V si haberem, darem desaparece al verse sustituido por si habuissem, dedissem (véase a este respecto, p. ej., Grandgent, 1928: 95-96 y Bourciez, 1946 [1910]: 129-130). Como consecuencia, en las oraciones condicionales latinas surge nueva oposición modal:

reales (indicativo: si habeo, do) vs. no reales (subjuntivo: $s i$ habuissem, dedissem).

Por otro lado, es de subrayar que ya en la época arcaica (Plauto) el indicativo aparecía también en los esquemas condicionales no reales. Se trataba del empleo del imperfecto y del 
pluscuamperfecto de indicativo, sobre todo en la apódosis condicional. Este uso fue iniciado, como solía ocurrir, por los verbos modales (possum, debeo, necesse est) y se hizo más común en los textos clásicos:

(16) et, si fata deum, si mens non laeva fuisset, impulerat ferro Argolicas foedare latebras (Virg.) (Nowikow, 1995: 169).

Como resultado, en la época posclásica, al lado del esquema no real canónico con el pluscuamperfecto de subjuntivo si habuissem, dedissem, se daban varios esquemas no reales periféricos con los tiempos imperfecto y pluscuamperfecto de indicativo: $s i$ habuissem, dabam; si habebam, dabam; si habuissem, dederam; si habueram, dederam. Además, en la época posclásica aumentó la frecuencia del esquema si habuissem, daturus eram que se daba ya en el periodo arcaico:

(17) si tacuisset, tamen ego eram dicturus (Plauto) (Nowikow, 1995: 170).

Precisamente la forma daturus eram fue reemplazada por la nueva formación dare habebam (habui) creándose, de esta manera, el futuro condicional sintético romance (cfr. el consabido ejemplo sanare te habebat Deus...) que formó parte del nuevo esquema condicional no real si habuissem, dare habebam.

Al concluir, cabe señalar que ya en latín se daban dos tipos de modalización que en los esquemas condicionales no reales se llevaban a cabo

a) mediante el pluscuamperfecto de subjuntivo cantavissem y

b) a través del uso de las formas temporalmente desplazadas de indicativo cantabam, cantaveram y de la nueva formación cantare habebam (esp. cantaría).

\section{5.}

\section{Los esquemas condicionales en las lenguas románicas}

Como es sabido, los sistemas verbales de los romances se caracterizaban por varias innovaciones importantes tales como, por ejemplo, la creación de los tiempos compuestos según el modelo 
habere + participio (habeo cantatum). De esta manera en los esquemas condicionales españoles no reales entran las formas había / habría / hubiese / hubiera cantado ${ }^{2}$. Por supuesto, igual que en latín, hay que distinguir entre esquemas canónicos (nucleares) y periféricos (marginales).

Partiendo de los tiempos verbales simples procedentes del latín se distinguen tres grupos principales con los siguientes condicionantes (prótasis) en los esquemas canónicos (cfr. Nowikow, 1995: 171):

a) $s i+$ imperfecto de subjuntivo (modelo si habuissem);

b) $s i+$ la forma en -ra (modelo si habueram);

c) $s i$ + imperfecto de indicativo (modelo si habebam).

En el condicionado (apódosis) de todos los esquemas canónicos se emplea la forma procedente de cantare habebam (p.ej., esp. cantaría).

\section{6.}

\section{El modelo 'si habuissem'}

La mayoría de las lenguas románicas conserva este modelo (cfr., p. ej., Gamillsheg, 1970; Coseriu, 1976; Nowikow, 1995; Bermejo Calleja, 2008: 221-222, 225):

- español: si tuviese, daría;

- gallego: si tuvese, daría;

- portugués: si tivesse, daria;

- catalán: si tingués, daria;

- provenzal: s'aguessi, donarià;

- italiano: si avessi, darei.

2 Sobre la diacronía y sincronía de los esquemas condicionales en español véase Mendeloff, 1959; Rojo, Montero Cartelle, 1983; Montero Cartelle, 1989; Söhrman, 1991; Veiga, 1991; Nowikow, 1993; Porcar Miralles, 1993; Montolío, 1999; Veiga, Mosteiro Louzao, 2006. Cabe recordar que en los últimos años han aparecido varios trabajos de Bartol Hernández p. ej., 2012, 2016, 2019; dedicados a diferentes aspectos de la evolución histórica de las oraciones condicionales castellanas. 
En francés el esquema en cuestión se daba en los textos antiguos conservándose, esporádicamente, hasta los siglos XV-XVI. En cambio, en catalán y provenzal el modelo si habuissem existe al lado del de si habebam.

El gallego al conservar este esquema, lo emplea no solo con valor de simultaneidad / posterioridad sino con el de anterioridad (el gallego no tiene formas compuestas del tipo haber + participio y mantiene el pluscuamperfecto sintético procedente del latín cantaveram). Por otro lado, en gallego cantara puede funcionar como alomorfo subjuntivo de cantase y equivale a los tiempos castellanos hubiera / hubiese cantado.

\section{7. \\ El modelo 'si habueram'}

Este modelo es claramente minoritario dentro de los romances actuales. Así, el español, el gallego y el valenciano son las únicas lenguas que lo continúan (véase, entre otros, Ridruejo, 1985; Veiga, 1986, 1996; Nowikow, 1995, 2011; Luquet, 2004; en el último libro destaca la segunda parte [99-194] dedicada casi exclusivamente a la forma en $-r a$ ). En castellano este esquema funciona con valor temporal primario de simultaneidad / posterioridad. La misma situación se observa en el gallego que, sin embargo, utiliza el modelo en cuestión también con vector temporal de anterioridad. En cambio el valenciano mantiene el esquema si tinguera, daria que equivale al castellano si tuviera, daría.

En los demás romances el modelo si habueram despareció muy pronto al perderse la forma de pluscuamperfecto latina o, en el caso de algunas lenguas, se daba, esporádicamente, en la época medieval (cfr. Nowikow, 1995: 172-173). Así, en el portugués antiguo se registraban ejemplos tales como

(18) Se o desejo fizera a jornada, já lá estivera

que actualmente, siendo empleos literarios y arcaizantes, son periféricos.

En cambio, en el provenzal las formas de tipo aguèra, fora se utilizaban en las condicionales hasta el siglo XVII: 
(19) Si lor aguèra lors obras corregidas, totis aurián badat quand las aurián legidas (Galhard).

Los usos se daban preferentemente en la apódosis o en las cláusulas independientes:

(20) Si vos fossáz del mún, lo múnz améra zo que era só (Évangile de Saint Jean).

(21) Mai me valgra quem agues mort (L'Évangile de l'enfance).

El mismo fenómeno tuvo lugar en el catalán medieval:

(22) El diable pogra cegar si's volgés (Homilies d'Organyà).

Por su parte, en francés el pluscuamperfecto de indicativo en -ra se perdió muy pronto dándose los últimos empleos de cantaveram en los siglos IX-X mientras que en el italiano antiguo dicha forma se empleaba muy esporádicamente.

\section{8.}

\section{El modelo 'si habebam'}

Este modelo funciona como esquema canónico en francés ( $s i$ j'avais, je donnerais), provenzal (s'aviá, donariá) y catalán (si tenia, daria) (véase también Nowikow, 1995: 173). Cabe subrayar que en francés es esquema único con valor de no realidad simultanéa o posterior. En cambio en el provenzal s'aviá aparece al lado de s'aguéssi (cfr. el modelo si habuissem). Por su parte, en catalán si tenia, daria se recomienda como uso normativamente más correcto que si tingués, daria.

A diferencia de tres lenguas mencionadas supra, en español, gallego, portugués e italiano las formas del imperfecto de indicativo se emplean en la prótasis y/o apódosis condicionales de manera más periférica, es decir, en general, más limitada, matizada diafásica y diastráticamente, etc. (cfr., p. ej., Nowikow, 1992; Bermejo Calleja, 2008: 226, 246-247, 253). 


\section{9. \\ Observaciones finales: el modo subjuntivo y el desplazamiento temporal en las condicionales latinas y romances}

Al concluir, quisiéramos presentar nuestras observaciones sobre la influencia de factores tales como el empleo del modo subjuntivo y el desplazamiento temporal en la modalización del contenido proposicional y en la conformación de los esquemas condicionales no reales en latín y en las lenguas románicas. De modo que hemos sacado las siguientes conclusiones:

1. En latín la modalización de la relación entre condición y consecuencia con valor de no realidad se llevaba a cabo, fundamentalmente, mediante el modo subjuntivo (pluscuamperfecto cantavissem). El esquema "canónico" para la expresión de anterioridad, y después al desaparecer si haberem, para los vectores temporales de simultaneidad / posterioridad, era si habuissem, dedissem.

2. En cambio, los esquemas no reales "periféricos" se construían con el imperfecto y con el pluscuamperfecto de indicativo: $s i$ habuissem, dabam; si habebam, dabam; si habuissem, dederam; si habueram, dederam. Esto quiere decir que en tales casos la modalización se realizaba mediante el mecanismo de desplazamiento temporal.

3. En las lenguas románicas se amplía la zona distribucional del indicativo, lo que se lleva a cabo, básicamente, mediante tres procedimientos relacionados con el desplazamiento temporal:

- la creación del nuevo 'condicional' románico cantare habebam > esp. cantaría empleado en la apódosis;

- la difusión del modelo si habebam en los esquemas "canónicos" del francés (sij'avais, je donnerais), provenzal (s’aviá, donariá) y catalán (si tenia, daria);

- la penetración del imperfecto de indicativo en la apódosis condicional de los esquemas "periféricos", p. ej., esp. si tuviese, daba (por el "canónico" daría). 
4. Sin embargo, muchas lenguas romances mantienen la modalización mediante el modo subjuntivo. Por ejemplo, el español, el gallego, el portugués y el italiano lo hacen a través de los esquemas "canónicos", mientras que el provenzal y el catalán aplican este mecanismo en los modelos "periféricos".

5. Lenguas románicas tales como español, gallego y valenciano continúan el modelo del latín posclásico con el pluscuamperfecto de indicativo si habueram. No obstante, hay una particularidad importante. La forma en - $r a$ sufre dos cambios fundamentales. Por un lado, se trata de la llamada "subjuntivización" de la forma en -ra y, por otro, de la "posteriorización" temporal de esta última al comenzar a expresar las relaciones de simultaneidad / posterioridad en vez de la anterioridad. La única excepción en este caso es el gallego (cfr. el modelo si habueram).

En resumen, podríamos formular tres observaciones tipológicas principales sobre el mecanismo de modalización en los esquemas condicionales no reales en el proceso de transición del latín a los romances:

- Las llamadas lenguas galorrománicas (en primer lugar francés y provenzal) representan la modalización realizada, exclusiva o básicamente, a través del mecanismo de desplazamiento temporal de los tiempos del modo indicativo. La misma opción la escoge el catalán definido a veces como lengua puente entre los romances galo e iberorrománicos.

- Las lenguas iberorrománicas grosso modo mantienen como recurso modalizador principal el modo subjuntivo, lo que se refiere, en primer lugar, al español, gallego y portugués. Algunas lenguas (español, gallego, valenciano) incluso amplían la zona del subjuntivo al "subjuntivizar" las formas procedentes del pluscuamperfecto de indicativo latino cantaveram.

- El italiano al conservar el modelo si habuissem (si avessi, darei) y al perder la forma cantaveram ocupa una posición intermedia entre los idiomas galo e iberorrománicos.

- En comparación con el latín, en su conjunto, las lenguas románicas demuestran mayor predisposición hacia la modalización del contenido proposicional mediante el 
desplazamiento temporal de los tiempos del modo indicativo.

- Sin embargo, con respecto a los mecanismos de modalización, dentro de los romances se distinguen dos tipos de lenguas. Los romances galorrománicos reducen la influencia del modo subjuntivo y aumentan el papel del deplazamiento temporal de los tiempos del modo indicativo (cfr. el modelo si habebam). En cambio, las lenguas iberorrománicas se presentan como herederos más fieles del latín al mantener la participación activa del modo subjuntivo (cfr. el modelo si habuissem) e incluso al incorporar la forma originariamente indicativa cantaveram (pluscuamperfecto de indicativo) en el subsistema del modo subjuntivo (cfr. el imperfecto de subjuntivo español cantara procedente de cantaveram).

\section{Bibliografía}

Altieri Megale, A. (1988): Gramática latina. Puebla: Universidad Autónoma de Puebla.

Bartol Hernández, J.A. (2012): "Habría dado con valor DEDISSEM. Siglos XVI-XVIII”, en: T. Jiménez Juliá, B. López Meirama, V. Vázquez Rozas, A. Veiga (eds.), Cum corde et in nova grammatica. Estudios ofrecidos a Guillermo Rojo. Santiago de Compostela: Universidade de Santiago de Compostela, Servizo de Publicacións e Intercambio Científico: 53-64.

Bartol Hernández, J.A. (2016): "La consolidación de la forma habría dado con valor dedissem. Su uso en la apódosis condicional irreal en la segunda mitad del XIX", en: A. López Serena, A. Narbona Jiménez, S. del Rey Quesada (eds.), El español a través del tiempo. Estudios ofrecidos a Rafael Cano Aguilar. Sevilla: Editorial Universidad de Sevilla, Vol. 1: 369-384.

Bartol Hernández, J.A. (2019): “De (en) otra guisa con valor procondicional”, en: A. Briz, M. a J. Martínez Alcalde, N. Mendizábal, M. Fuertes Gutiérrez, J.L. Blas, M. Porcar, Estudios lingüísticos en homenaje a Emilio Ridruejo. Valencia: Publicacions de la Universitat de València, Vol. 1: 59-69.

Bassols de Climent, M. (1976): Sintaxis latina. Madrid: CSIC. 
Bermejo Calleja, F. (2008): Le subordinate avverbiali. Uno studio contrastivo spagnolo-italiano. Bologna: Bononia University Press.

Bourciez, É. (1946 [1910]): Éléments de linguistique romane. Paris: Librairie C. Klincksieck.

Coseriu, E. (1976): Das romanische Verbalsystem. Tübingen: T.B.L.

Gamillscheg, E. (1970): Studien zur Vorgeschichte einer romanischen Tempuslehre. Tübingen: T.B.L.

Grandgent, C.E. (1928): Introducción al latín vulgar. Madrid: Publicaciones de la Revista de Filología Española.

Luquet, G. (2004): La teoría de los modos en la descripción del verbo español (un nuevo planteamiento). Madrid: Arco / Libros.

Mendeloff, H. (1960): The Evolution of the Conditional Sentence Contrary to Fact in Old Spanish. Washington: The Catholic University of America Press.

Montero Cartelle, E. (1989): Gonzalo de Berceo y El Libro de Alexandre. Aproximación al sistema verbal de la época desde los esquemas condicionales, "Verba", Anexo 30. Santiago de Compostela: Universidade de Santiago de Compostela.

Montolío, E. (1999): "Las construcciones condicionales", en: I. Bosque, V. Demonte (eds.), Gramática descriptiva de la lengua española, Vol. 3. Madrid: RAE, Espasa-Calpe: 3643-3737.

Nowikow, W. (1991): “Observaciones morfosintácticas sobre el condicional eslavo y románico (aspecto diacrónico y sincrónico)". Anuario de Lingüística Hispánica, VII: 407-413.

Nowikow, W. (1992): "Sobre el imperfecto de indicativo en la apódosis de las condicionales”, Estudios Hispánicos, II: 47-62.

Nowikow, W. (1993): Evolución funcional de los esquemas condicionales no reales en el español de los Siglos de Oro. Łódź: Wydawnictwo Uniwersytetu Łódzkiego, Frankfurt: Vervuert Verlag.

Nowikow, W. (1995): “Evolución tipológica de los esquemas condicionales no reales en las lenguas romances". Studia Romanica Posnaniensia, 20: 167-176.

Nowikow, W. (2011): "Sobre el esquema condicional más plurifuncional en la historia del castellano: en torno a la interpretación 
del cambio de si tuviera, diera en los siglos XVI-XVII", en: C. Sinner, J.L. Ramírez Luengo, M. ${ }^{\mathrm{a}}$ J. Torrens Álvarez (eds.) Tiempo, espacio y relaciones espacio-temporales desde la perspectiva de la lingüística histórica, Instituto Historia de la Lengua, dir. José A. Pascual, Monografías, X. San Millán de la Cogolla: Cilengua.

Nowikow, W. (2013): "Sobre la modalización del contenido proposicional: Contraste tipológico entre lenguas románicas y eslavas", en: A. Pamies Bertrán (ed.), De lingüística, traducción y léxico-fraseología. Homenaje a Juan de Dios Luque Durán. Granada: Editorial Comares, 65-72.

Nowikow, W. (2017): "Modos verbales", en: W. Nowikow (ed.), Gramática contrastiva español-polaco, Colección "Manufactura Hispánica Lodziense”, 2. Łódź: Wydawnictwo Uniwersytetu Łódzkiego.

Nowikow, W. (2018): "La comparación de los sistemas modotemporales español y polaco como modelo de análisis comparado de características tipológico-funcionales de las lenguas románicas occidentales y eslavas norteñas", en: E. Hernández Socas, J.J. Bautista Rodríguez, C. Sinner (eds.), Clases y categorías lingüísticas en contraste. Español y otras lenguas, Studien zur romanischen Sprachwissenschaft und interkulturellen Kommunikation, Herausgegeben von Gerd Wotjak, Band 124. Berlin-Bern-Bruxelles-New York-Oxford-Warszawa-Wien: Peter Lang: $97-115$.

Nowikow, W. (2018) : "Sobre metáforas gramaticales: el caso de los tiempos verbales”, en: Pamies, I.M. ${ }^{a}$ Balsas, A. Magdalena (eds.), Lenguaje figurado y competencia interlingüística (I). Aspectos teóricos. Granada: Editorial Comares: 77-85.

Nutting, H.C. (1926): The Latin Conditional Sentence. "Classical Philology", 8: 1-185.

Porcar Miralles, M. (1993): La oración condicional: la evolución de los esquemas verbales condicionales desde el latín al español actual. Castelló: Publicacions de la Universitat Jaume I.

Ridruejo, E. (1985): "La forma verbal en -ra en valenciano". Actes du XIIéme Congrès International de Linguistique et Philologie Romanes, 2. Université de Provence: 437-448. 
Rojo, G. (1974): “La temporalidad verbal en español”. Verba, 1: 68-149.

Rojo, G., Montero Cartelle, E. (1983): La evolución de los esquemas condicionales (Potenciales e irreales desde el poema del Cid hasta 1400). "Verba", Anejo 22. Santiago de Compostela: Universidad de Santiago de Compostela.

Rojo, G., Veiga, A. (1999): "El tiempo verbal. Los tiempos simples”, en: I. Bosque, V. Demonte (eds.), Gramática descriptiva de la lengua española. Madrid: RAE, Espasa-Calpe, Vol. 2: 2867-2934.

Safarewicz, J. (1950): Gramatyka historyczna języka łacińskiego. Część II. Składnia. Warszawa: PZWS.

Söhrman, I. (1991): Las construcciones condicionales en castellano contemporáneo. "Acta Universitatis Upsaliensis", Uppsala.

Stefański, W. (1987): La proposition conditionnelle dans les langues indo-européennes. Poznań: Wydawnictwo Naukowe UAM.

Veiga, A. (1986): "Verbo latino e verbo galego. Notas para unha análise comparativa”. Verba, 14: 169-216.

Veiga, A. (1991): Condicionales, concesivas y modo verbal en espanol, "Verba", Anexo 34. Santiago de Compostela: Universidade de Santiago de Compostela.

Veiga, A. (1996): La forma verbal española cantara en su diacronía, Colección Lucus Lingua, 2. Santiago de Compostela: Universidade de Santiago de Compostela, Servicio de Publicacións.

Veiga, A. (2012): "Sobre el concepto de dislocación en la teoría temporal de G. Rojo”, en: T. Jiménez Juliá, B. López Meirama, V. Vázquez Rozas, A. Veiga (eds.), Cum corde et in nova grammatica. Estudios ofrecidos a Guillermo Rojo. Santiago de Compostela: Universidade de Santiago de Compostela, Servizo de Publicacións e Intercambio Científico: 855-866.

Veiga, A., Mosteiro Louzao, M. (2006): El modo verbal en cláusulas condicionales, causales, consecutivas, concesivas, finales y adverbiales de lugar, tiempo y modo. Salamanca: Ediciones Universidad. 